\title{
eJRIEPS
}

Ejournal de la recherche sur l'intervention en éducation physique et sport

22 | 2011

Varia

\section{La rentabilité des traits " masculins » dans un lycée favorisé, en particulier en EPS}

\section{Julien Moniotte}

\section{Q OpenEdition}

\section{Journals}

Édition électronique

URL : http://journals.openedition.org/ejrieps/4646

DOI : 10.4000 /ejrieps.4646

ISSN : 2105-0821

Éditeur

ELLIADD

\section{Référence électronique}

Julien Moniotte, "La rentabilité des traits « masculins » dans un lycée favorisé, en particulier en EPS », eJRIEPS [En ligne], 22 | 2011, mis en ligne le 01 janvier 2011, consulté le 27 février 2020. URL : http:// journals.openedition.org/ejrieps/4646; DOI : 10.4000/ejrieps.4646

\section{(c) (1)}

La revue eJRIEPS est mise à disposition selon les termes de la Creative Commons Attribution 4.0 International License. 


\section{La rentabilité des traits « masculins » dans un lycée favorisé, en particulier en EPS}

Julien Moniotte

LASELDI (LAboratoire de SEmio-Linguistique, Didactique et Informatique - EA 02281)

\section{Résumé}

Cet article présente des résultats issus de notre thèse de doctorat. En nous appuyant sur le paradigme de l'androgynie psychologique (Bem, 1978), la sociologie du curriculum (Forquin, 1989) et les théories de la reproduction (Bourdieu \& Passeron, 1970), nous analysons l'effet de l'orientation de genre et du sexe sur le jugement professoral (les notes et les appréciations sur les bulletins trimestriels). Les concepts de genre, de sexe, du masculin et du féminin, sont définis, puis l'ancrage de l'EPS dans les institutions scolaire et sportive est rappelé. Ensuite, les résultats sont présentés et commentés. Le genre masculin apparaît comme favorisé en EPS, mais également dans l'ensemble de l'institution scolaire.

\section{Introduction}

La compétition scolaire est favorable aux filles (Felouzis, 1993). Nous pouvons penser que le genre féminin - dont théoriquement les filles sont plus proches - est favorisé à l'Ecole. Cependant, cette dernière héberge une discipline d'enseignement à part, puisqu'en EPS, l'écart moyen entre les garçons et les filles est au bénéfice des premiers. II semblerait donc que l'EPS avantage le genre masculin. Dès lors, la question suivante apparaît : quels élèves l'Ecole et l'EPS font-elles réussir? A priori, pas les mêmes sachant que les filles, dominant la compétition scolaire, subissent l'hégémonie des garçons en EPS. Pour autant, certaines filles réussissent en EPS et une part importante de garçons connait une très bonne scolarité. II est possible que la culture transmise au sein de l'Ecole et particulièrement en EPS soit proche de la culture dominante d'un même groupe social. Dans ce cas, il pourrait y avoir un effet loupe de l'EPS. Celle-ci nous dévoilerait certains phénomènes beaucoup plus difficiles à déceler concernant l'institution scolaire comme par exemple, l'existence d'un curriculum caché masculiniste. Forquin (1989, p. 23) définit le curriculum caché comme "des choses qui s'acquièrent à l'école (savoirs, compétences, représentations, rôles, valeurs) sans jamais figurer dans les programmes officiels ou explicites, soit parce qu'elles relèvent d'une «programmation idéologique » d'autant plus 


\section{eJRIEPS 22 janvier 2011}

impérieuse qu'elle est plus occulte [...], soit parce qu'elles échappent au contraire à tout contrôle institutionnel et se cristallisent comme des savoirs pratiques, des recettes de « débrouillardise » ou des valeurs de contestation fleurissant dans les interstices ou les zones d'ombre du curriculum officiel ».

Mosconi $(1994,1995,2004)$ a dénoncé le curriculum caché masculiniste de l'Ecole, et a souligné le «masculin neutre - le déni des différences sexuelles et la violence symbolique dont sont victimes les filles, par la prédominance des valeurs et du pouvoir masculin - qui y réside. Kelly (1987, citée par Duru-Bellat, 2004b) note que les enseignants, plutôt centrés sur les inégalités sociales, considèrent l'élève comme asexué, ignorant l'existence d'inégalités inter-sexes.

Cependant, ces inégalités sont présentes à l'Ecole comme dans la société, se dissimulant jusque dans les petites choses du quotidien. Selon Goffman (2002, p.82), « la ségrégation des toilettes est présentée comme une conséquence naturelle de la différence entre les classes sexuelles, alors qu'en fait c'est plutôt un moyen d'honorer, sinon de produire, cette différence ». II serait plus exact de parler de discriminations que de différences. La signalétique des portes de toilettes peut servir à justifier certaines injustices. Yaël Nazé (2006) rapporte l'anecdote suivante : en 1965, Vera Cooper Rubin est à l'origine d'une révolution copernicienne. Elle est la première femme à laquelle on accorde le droit d'observer « en toute légalité » à l'observatoire du Mont Palomar. Toutes les astronomes qui en avaient fait la demande auparavant s'y étaient vu refuser l'accès car, « à cause de la délimitation des commodités, il n'est pas possible d'accepter les demandes provenant des femmes ». II n'existe alors qu'une seule toilette, pour hommes, dans le bâtiment. Cet argument se réclamant d'une évidence pratique, d'un certain bon sens, sert en fait à dissimuler le refus fait aux femmes d'accéder à l'astronomie et plus largement à la science. Il permet aussi de clore le débat, d'empêcher la réflexion sur les inégalités subies par les femmes et les filles. Celle-ci mène à réinterroger le doublon masculin/féminin et donc le(s) genre(s). Ce dernier constitue une variable centrale dans notre étude qui s'appuie sur l'androgynie psychologique (Bem, 1978).

L'article présente une revue de littérature, la problématique et des hypothèses, la méthodologie et enfin des résultats issus de notre travail de thèse de doctorat.

\section{Revue de questions}

Nous débutons par la présentation des concepts renvoyant au genre, au sexe, au masculin et au féminin. Leur polysémie oblige à les définir afin de rendre compréhensible 


\section{eJRIEPS 22 janvier 2011}

les discussions. Ces dernières amènent à soulever rapidement la problématique du genre dans le sport. L'institution sportive joue en effet un rôle prépondérant dans la construction de la masculinité, mais influe également en partie sur la culture corporelle transmise en EPS. Aussi, les travaux centrés sur l'institution scolaire puis sur l'EPS seront exposés.

L'EPS étant traversée par deux cultures institutionnelles (scolaires et sportives), il paraît nécessaire de les étudier pour produire une analyse féconde des résultats. Si la domination de la culture scolaire semble avérée en EPS, il n'en demeure pas moins que la culture corporelle qui y est prépondérante est celle de la culture sportive. Bien que l'EPS soit intégrée au sein de l'éducation nationale depuis plusieurs décennies, les enseignants se caractérisent encore par leur proximité avec la pratique fédérale en tant qu'entraîneurs, dirigeants ou (anciens) sportifs (MEN, 2007). D'un côté, l'EPS ne vise pas l'émergence d'une élite mais la réussite de tous, d'un autre, le maintien de la «logique interne » de chaque APSA reste une préoccupation récurrente. Par ailleurs, le poids des pratiques fédérales des élèves sur leur réussite en EPS est une question perpétuellement débattue.

1. 1. Le genre, une autre façon de parler du masculin et du féminin

Le genre est l'ensemble des attributs psychologiques, activités, rôles et statuts sociaux culturellement assignés à chacune des catégories de sexe et constituant un système de croyances, dont le pivot est le principe d'une détermination biologique (Hurtig, Kail \& Rouch, 2002). Il a trait non à la différence, mais à la différenciation sociale des sexes (Mathieu, 2002). La polysémie du mot « sexe » renvoie à la fois aux organes sexuels et à une identité psycho-sociale - masculin/féminin - des individus (Vinet, 2008).

Marro et Vouillot (2004) préfèrent le terme de rapports sociaux de sexe à celui de genre pour mettre en avant les relations de domination des hommes sur les femmes. En outre, selon Butler (1990, citée par Vidal, 2008), « homme » et «femme » ne désignent nullement une identité naturelle, ce sont des performatifs. Leur énonciation est l'accomplissement d'un acte (Laugier, 2004). Par conséquent, «l'essence naturelle » du féminin et du masculin n'est qu'une mystification langagière (Vidal, 2008).

Pour Constantinople (1973), les deux présupposés utilisés pour représenter masculinité et féminité, qui sont l'unidimensionnalité et la bipolarité, sont invalides. Elle démontre que ce sont deux dimensions distinctes et indépendantes l'une de l'autre. Chaque individu peut à la fois endosser des traits et rôles «féminins » et d'autres « masculins », par exemple se comporter en leader et être attentif aux autres. Cependant, endosser les traits masculins peut parfois poser problème pour une femme. Par exemple, le fait d'être femme et sportive constitue un défi aux rôles sociaux prescrits et nécessite une mise en scène particulière 
eJRIEPS 22 janvier 2011

de soi, une représentation d'un rôle dans l'espace sportif sans prendre le risque de s'exposer à un conflit de normes (Lefèvre, 2005). «La sportive faite homme, disgraciée, dévaloriserait l'image de la femme, comme s'il existait un féminin pur, originel. Le sport devient un miroir grossissant des travers de l'affranchissement des femmes » (Baillette \& Liotard, 1999, p. 49). L'image de la sportive rentrerait en contradiction avec les modèles corporels de la féminité. La participation des filles pendant les séances d'une EPS « sportivisée » pourrait dès lors poser des problèmes identitaires.

Avant d'être sportive, l'EPS est d'abord une discipline scolaire. Un détour par son institution de tutelle, l'Ecole, semble nécessaire. Des recherches sur le jugement professoral, en particulier sur l'évaluation, puis des travaux sur la mixité scolaire sont présentées dans les paragraphes suivants.

1. 2. L'Ecole, productrice et/ou reproductrice des inégalités?

Évaluer c'est créer des hiérarchies d'excellence, qui serviront de base à la progression dans le cursus scolaire, l'orientation, la certification et bien souvent l'embauche (Perrenoud, 1998). Ces hiérarchies renseignent plus sur la position d'un élève par rapport aux autres. Pour Merle (1996), classer les élèves est une des significations forte de l'activité enseignante, et l'utilisation de l'expression « jugement professoral » ne fait que le rappeler. Ce jugement désigne les notes attribuées, mais aussi les appréciations sur les bulletins scolaires, les discussions sur les compétences de l'élève en conseil de classe et aux délibérations du bac (Merle). Perrenoud (1998) rapporte que les pratiques d'évaluation, sans engendrer des inégalités dans la maîtrise des savoirs et savoir-faire, sont cruciales dans leur transformation en classement et jugements scolaires.

Par conséquent, l'évaluation est négociée pour son impact sur la sélection-orientation, elle s'inscrit toujours dans un rapport social, une transaction plus ou moins tendue entre l'enseignant d'un côté, l'élève et sa famille de l'autre (Perrenoud, 1998). Merle (1996) parle d'arrangement évaluatif, c'est-à-dire le produit d'interactions multiples et de considérations diverses : maintenir un minimum d'ordre scolaire, faire progresser les élèves, favoriser leur intégration scolaire, respecter les normes de la discipline et de l'établissement. L'élève type, rendant une copie type, corrigée par un barème et un enseignant types est une croyance scolaire: tout jugement professoral émerge dans un contexte social donné (Merle, 1996). Ce jugement a généralement valeur de sanction, il équivaut à une énonciation « performative » plutôt que « constatative » (Forquin, 1989).

Le jugement professoral ne se réduit nullement aux notes distribuées. Ces dernières sont complétées par les appréciations portées sur les bulletins ou les copies. Le bulletin 


\section{eJRIEPS 22 janvier 2011}

scolaire est un instrument qui relie trois pôles : famille, école, évaluation (Sarrazy, 2000). II évalue la distance aux exigences du curriculum caché, non au curriculum effectif (Sarrazy, 2000). D'ailleurs, Merle (1996) note que les comportements en classe peuvent transparaître dans les appréciations sur les bulletins. Une enquête de Terrail (1995, cité par Merle) montre qu'à compétences égales à des tests standards, les filles obtiennent en moyenne 0,78 point de plus que les garçons. L'application et le sérieux des filles seraient récompensés. Les travaux de Felouzis (1994) expliquent leur meilleure réussite par un investissement plus important, une plus forte adhésion aux valeurs scolaires et la demande de l'Ecole de compétences plus proches du modèle féminin.

Cependant, Mosconi (1994) dénonce le « masculin neutre » régnant à l'Ecole. D'ailleurs, si la socialisation des filles est payante à court terme, Duru-Bellat (2004a) souligne que les inégalités sont différées et apparaissent plus tard dans les phases intenses d'orientation. Plusieurs recherches sur ces inégalités ont été menées. Duru-Bellat (1994, 1995a) en fait part dans une revue de questions sur les choix professionnels et l'anticipation de l'avenir. Ces travaux montrent que les choix d'orientation des élèves répondent à une certaine « logique de sexe »; filles et garçons ont bien intégré que les métiers les plus prestigieux sont des métiers «masculins». Par conséquent, à niveau scolaire égal, les filles ont tendance à s'orienter vers des études menant à des métiers moins prestigieux sans que conseillers pédagogiques et conseils de classe n'y trouvent à redire. Duru-Bellat (2004a) parle alors de l'Ecole comme «agence d'orientation» reproduisant les flux sexués. Néanmoins, la chercheure remarque que ce n'est pas l'Ecole qui décide que certains métiers sont quasiment inaccessibles aux femmes et que les places scolaires et sociales les plus favorables sont limitées. Le choix de nombreuses filles de suivre les filières féminisées répondrait donc à une stratégie d'anticipation de l'avenir professionnel. Ces choix moins ambitieux sont à insérer dans un environnement plus ou moins contraignant. Ce manque d'ambition peut également provenir de l'intériorisation d'un programme latent. Selon Sadker, Sadker et Long (1993), un curriculum caché est créé par l'omission des femmes dans les livres scolaires. Les élèves apprennent alors implicitement que les femmes sont moins importantes que les hommes. Le curriculum caché se manifeste de bien des manières. Par exemple, la réaction des enseignants face aux comportements en classe diffère en fonction du sexe des élèves. Le maintien de l'ordre oblige les enseignants à se centrer sur les garçons « naturellement » plus enclins au chahut, en particulier physique, qui n'est accepté que comme exclusivement « masculin ». Dès que le physique intervient, l'ordre biologique revient. L'EPS n'y échappe pas. Robinson (1992, 


\section{eJRIEPS 22 janvier 2011}

cité par Duru-Bellat, 1995b) note qu'en cours d'EPS certains enseignants laissent transparaître leurs représentations en invitant les garçons à ne pas se rabaisser en se conduisant comme des filles, ces dernières représentant le modèle négatif. Nous nous intéressons maintenant à cette discipline où les filles connaissent, en moyenne, plus de difficultés que les garçons.

1. 3. L'EPS, le poids de l'institution sportive

Depuis la V $V^{e}$ République, l'éducation physique s'est « sportivisée » - domination du sport sur les autres disciplines corporelles (Caillat, 2002). D'ailleurs, Terret, Cogérino et Rogowski (2006) soulignent que si l'institution scolaire, à travers l'EPS, a joué un rôle dans la féminisation du sport des années 1970, en se sportivisant, elle a hérité de la séparation des corps (sexués) et de la domination masculine, légitimant la division des sexes qui s'estompe parallèlement dans le milieu scolaire.

Au bac, David (2000) constate un écart inter-sexes moyen de 1,1 point en EPS. Davisse et Louveau (2003) remarquent que cet écart est réduit dans la filière scientifique, alors que l'amplitude entre filles des filières scientifiques et filles des filières tertiaires est bien plus importante. Les statistiques indiquent que les filles - en particulier celles issues des milieux défavorisés, très présentes dans les filières tertiaires - pratiquent beaucoup moins que les garçons une APS en dehors de l'EPS (Davisse \& Louveau, 2003). Un effet filière se combine à un effet pratiques extra-scolaires, souvent avancé pour expliquer le retard des filles en EPS. Les autres explications courantes sont :

- le recours fréquent aux sports, profondément marqués par des modèles sociaux masculins ;

- des interactions enseignant-élèves plus fréquentes pour les garçons ;

- des attentes différentes selon le sexe de l'élève en EPS.

Selon Chalabaev (2006), les stéréotypes de sexe dans les APS peuvent influencer la réussite des individus de diverses manières; par les biais perceptifs et les prophéties auto-réalisatrices produits par les professeurs (ils s'attendraient plus facilement, par un mélange de croyances et d'expériences personnelles, à de meilleures performances des garçons), par le poids des stéréotypes sur les élèves. Par ailleurs, Vigneron (2004) note d'après l'analyse des réponses qu'elle a obtenues par questionnaire auprès d'un millier de lycéens en terminale - qu'il n'est pas gênant pour les filles de se rapprocher du modèle féminin puisque la réussite en EPS est accessoire pour elles alors qu'elle est impérative pour l'identité masculine. Williamson (1996) souligne que les barèmes différenciés en éducation physique selon le sexe créent une pression sur les élèves ; les garçons doivent 


\section{eJRIEPS 22 janvier 2011}

répondre aux attentes sociales en surpassant les filles, alors que ces dernières sont censées ne pas atteindre le niveau des garçons. Selon Vigneron (2004), la participation " modérée » de certaines filles en EPS n'est pas tant subie que choisie. Une recherche de Cockburn et Clarke (2002, cités par Bienaimé-Patinet, 2009) remarque que certaines filles ressentent un "déficit de féminité » en EPS. Pour Combaz (1991), la culture transmise dans cette discipline ne correspond pas aux attentes des filles, pour qui la culture corporelle ne se résume pas à la culture sportive.

Les activités physiques et artistiques (APA) ont été introduites en EPS pour contrer la prééminence du sport, mais ont échoué à déconstruire l'ordre sexué (Ferez, 2004). Les APA, comme la mixité en EPS, n'assurent nullement l'égalité entre filles et garçons. Bienaimé-Patinet (2009) dénombre trois types de mixité, mais aucune n'est exempte de traitement différencié des élèves en fonction de leur sexe.

\section{Problématique et hypothèses}

En plus de l'appui sur l'androgynie psychologique (Bem, 1978), nous fondons nos travaux sur les sociologies du curriculum (Forquin, 1989) et de la reproduction (Bourdieu \& Passeron, 1970). La sociologie du curriculum pose le problème de la construction sociale des savoirs scolaires et permet de débattre de la légitimité de ce qui est enseigné et mérite de l'être (Poggi, 2000). Or, ouvrir ce débat, c'est déjà pointer le pouvoir de violence symbolique de la culture scolaire. De fait, nous pouvons rapprocher ces deux sociologies par leur contestation commune de l'ordre établi.

L'effet de deux variables sur le jugement professoral est analysé et les hypothèses suivantes sont éprouvées :

- les garçons et les élèves qui endossent les traits masculins obtiennent de meilleurs résultats que leurs camarades ;

- ils jouissent également d'appréciations professorales plus positives sur leurs bulletins.

\section{Méthodologie}

Les données ( $n=504$, dont 307 filles) ont été recueillies lors de l'année 2007-2008 dans un établissement favorisé (cf. tableau 1) ne représentant pas les établissements de son académie. Les CSP parentales de 19 filles et 7 garçons ne sont pas connues. 
eJRIEPS 22 janvier 2011

Tableau 1 : Origine sociale selon le sexe des élèves

\begin{tabular}{|l|l|l|l|}
\hline Origine sociale & Nombre de filles & Nombre de garçons & Total \\
\hline $\begin{array}{l}\text { un (deux) parent(s) cadre(s) ou } \\
\text { assimilé(s) }\end{array}$ & 122 & 93 & 215 \\
\hline $\begin{array}{l}\text { un (deux) parent(s) de profession } \\
\text { intermédiaire }\end{array}$ & 62 & 42 & 104 \\
\hline $\begin{array}{l}\text { un (deux) parent(s) employé(s) } \\
\text { un (deux) parent(s) ouvrier(s), ou en } \\
\text { situation précaire }\end{array}$ & 32 & 26 & 64 \\
\hline $\begin{array}{l}\text { un (deux) parent(s) artisan(s), } \\
\text { commerçant(s), agriculteur(s) }\end{array}$ & 34 & 12 & 44 \\
\hline Total & 288 & 17 & 51 \\
\hline
\end{tabular}

Les lycéens ont été soumis à un court questionnaire comportant un inventaire des rôles de sexe de Bem (IRSB). Ensuite, l'accès aux bulletins scolaires des lycéens a permis de prélever les moyennes et appréciations générales et celles en EPS. La version courte de Fontayne et al. (2000) a été choisie comme IRSB. Elle nous permet de classer les individus selon quatre orientations de genre. Les typés masculins (TM) endossent les traits et rôles «masculins » tout en rejetant les traits et rôles « féminins ». Les typés féminins (TF) endossent les traits et rôles « féminins» et rejettent les traits et rôles « masculins». Les androgynes (AND) endossent aussi bien les traits « masculins » que « féminins », alors que les non différenciés (ND) les rejettent. Notons que 5 garçons n'ont pas répondu à I'IRSB. 
eJRIEPS 22 janvier 2011

Tableau 2 : Orientation de genre selon le sexe des élèves

\begin{tabular}{|l|l|l|l|}
\hline Orientation de genre/sexe & filles & garçons & Total \\
\hline typé(e)s masculin(e)s & 84 & 48 & 132 \\
\hline androgyne(s) & 79 & 56 & 135 \\
\hline typé(e)s féminin(e)s & 73 & 42 & 115 \\
\hline non différencié(e)s & 71 & 46 & 117 \\
\hline Total & 307 & 192 & 499 \\
\hline
\end{tabular}

Pour traiter les appréciations, une variable fermée inspirée d'un travail de Sarrazy (2000) a été créée. Ce dernier consiste à classer les différents éléments des appréciations dans plusieurs catégories et sous-catégories.

Tableau 3 : Encodage des appréciations

\begin{tabular}{|l||l|}
\hline C1 & Appréciations relatives aux résultats de l'élève \\
\hline C2 & Appréciations relatives à une « morale scolaire » \\
\hline C3 & Jugement invoquant des qualités psycho-cognitives et motrices \\
\hline C4 & Injonctions ou « conseils » \\
\hline C5 & Attentes déçues ou potentiel détecté \\
\hline
\end{tabular}

\section{Résultats}

Deux grandes parties présentent les résultats, la première sur la notation, puis la seconde sur les appréciations portées sur les bulletins trimestriels. II semblerait que de nombreux biais agissent tant sur les notes que sur les appréciations. Le croisement de ces deux variables indiquerait les croyances et attentes du corps professoral.

\subsection{Dénotation et connotation à travers les notes}

II existe un lien statistique entre le sexe ou l'orientation de genre des lycéens et leurs notes en EPS. Le groupe des garçons a une moyenne de 14,36, celui des filles, 1,36 point de moins. Cet écart est statistiquement significatif, de même que les écarts inter-genres. Les TM ont une moyenne de 14,2, les AND, 14,07, les TF, 12,92, les ND, 12,67. Les 


\section{eJRIEPS 22 janvier 2011}

écarts inter-genres sont plus importants que les écarts inter-sexes. Les groupes des filles TM et AND ont des résultats proches voire légèrement supérieurs à ceux des groupes de garçons TF et ND.

Davisse (1999) rappelle que le sport - référence de l'EPS - est issu de l'histoire des hommes, répondant à une logique d'affrontement, de défi, d'épreuve. Ainsi, la culture corporelle transmise en EPS ne correspondrait pas aux attentes des filles (Combaz, 1991). Cependant, les résultats des filles TM et AND nous forcent à nuancer les propos de Combaz. II est plus exact de parler des attentes des filles les plus éloignées des modèles masculins. Fontayne et al. (2001) montrent que les TM et AND sont sur-représentés parmi les sportifs. Nous pouvons supposer que les TM et AND de notre échantillon sont plus proches de la culture sportive, ayant plus de chances de pratiquer une APS hors de l'EPS. Ces pratiques physiques extra-scolaires pourraient expliquer, en partie, la meilleure réussite des TM et AND.

En outre, si l'orientation de genre semble permettre de neutraliser l'effet du sexe pour les filles TM et AND, elle n'écrase nullement cette variable. A orientation de genre équivalente, les filles obtiennent des résultats inférieurs à ceux des garçons. Deux compétitions parallèles semblent coexister en EPS, au détriment des filles. Dans cette discipline, il est bien possible qu'elles soient pénalisées par les représentations professorales, ce qui semble confirmé par notre analyse des bulletins. Sexe et orientation de genre ont un effet significatif sur les moyennes obtenues en EPS, mais également sur les moyennes générales et les hiérarchies scolaires. Nous présentons les résultats statistiquement significatifs.

En plus d'obtenir une moyenne élevée en EPS, les garçons sont sur-représentés parmi l'élite et sous-représentés parmi les élèves obtenant des résultats médiocres dans cette discipline. Ils sont également sur-représentés parmi les lycéens dont les moyennes générales annuelles sont proches ou inférieures à $8 / 20$. Les filles, pour leur part, sont sous-représentées parmi l'élite en EPS. De plus, elles sont sur-représentées parmi les lycéens obtenant des moyennes générales médiocres. Notons que dans cette étude, filles et garçons obtiennent, en tant que groupes, une moyenne générale annuelle équivalente. Selon l'observation des moyennes générales, le sexe n'aurait aucune influence sur les résultats scolaires, contrairement à l'étude des répartitions des notes. Les garçons malgré leur sur-sélection - ont empiriquement plus de chances de se retrouver parmi les élèves « décrochés » - moyenne inférieure à 8/20. Les filles évitent ce « décrochage », mais se retrouvent plus facilement parmi les élèves aux résultats médiocres. Cette sur- 


\section{eJRIEPS 22 janvier 2011}

représentation des filles «médiocres» pourrait correspondre à une plus grande indulgence de la part des enseignants.

Le comportement scolaire de ces filles et les croyances du corps professoral («les filles travaillent plus que les garçons »), leur permettraient d'éviter la relégation parmi les lycéens au bas du classement scolaire. Inversement, les garçons en difficulté seraient plus facilement relégués. Cette croyance différentielle des efforts selon le sexe a plusieurs corollaires. Les garçons devraient leurs résultats à leur «talent », les filles à leur travail. Par conséquent, il pourrait aussi être plus difficile pour les filles de s'extraire de la « médiocrité ». En effet, si l'Ecole reconnaît le travail et l'effort, en «immunisant »les élèves méritant contre le décrochage, elle demanderait ce qu'elle ne transmet pas explicitement. Ainsi, les élèves trop scolaires ne pourraient prétendre à l'excellence. Ce qui permet d'atteindre celle-ci proviendrait de l'extérieur de l'Ecole. C'est également le cas en EPS, où les excellents se recrutent surtout parmi les sportifs pratiquants (Vigneron, 2004), élèves qui sont proches des modèles du masculin.

D'ailleurs, les TM ont une moyenne élevée en EPS et y sont sous-représentés parmi les élèves en échec. Ils sont également sur-représentés parmi les élèves obtenant les meilleures moyennes générales. De plus, ils ont une moyenne générale plus élevée que celle de l'ensemble des lycéens. Si ND et TF ont une faible moyenne en EPS, les premiers y sont sur-représentés parmi les élèves « en échec », alors que les seconds le sont parmi les élèves « médiocres ». En outre, les ND ont une faible moyenne générale et sont, sousreprésentés parmi l'élite scolaire, sur-représentés parmi les élèves « en échec ». Enfin, TF et AND ont des moyennes générales proches de celle de l'ensemble des lycéens étudiés. Ils représenteraient l'élève moyen. Au contraire, TM et ND correspondraient aux modèle et contre-modèle du bon élève. Ces interprétations semblent confirmées par l'analyse des appréciations trimestrielles.

4. 2. Appréciations, effet Pygmalion et dissonance cognitive?

Pour appréhender les attentes professorales, genre, appréciations et notes sont croisés. A appréciation équivalente - par exemple «assez bons résultats » -, certains groupes d'élèves obtiennent des résultats plus élevés - dans l'exemple les TM ont une moyenne de 13,17, les AND 12,95, les TF 12,31, les ND 12,17. II existerait de plus fortes exigences professorales envers les premiers groupes. D'après les différents croisements, en EPS, les enseignants attendraient plus des TM, puis des AND, qui y obtiennent d'ailleurs les meilleurs résultats. 
eJRIEPS 22 janvier 2011

II pourrait s'agir d'un effet Pygmalion (Rosenthal \& Jacobson, 1968, cités par Chalabaev, 2006). Les enseignants attendraient plus de certains lycéens. Ces plus fortes attentes faciliteraient leur propre réalisation et provoqueraient une meilleure réussite de ces élèves. Pour compléter cette analyse, nous observons les sur et sous-représentations issus du croisement genre/appréciations (cf. tableau 4).

Tableau 4 : Qualifications des résultats par les enseignants d'EPS (sous-catégories de C1) selon l'orientation de genre des élèves.

\begin{tabular}{|c|c|c|c|c|c|}
\hline 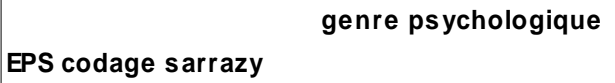 & masculin & androgyne & féminin & non-différencié & TOTAL \\
\hline excellence & $+9(30)$ & +9 (31) & $-10(8)$ & $-6(10)$ & $79(79)$ \\
\hline TB résultats & $+10(51)$ & $+6(48)$ & $-6(28)$ & $-8(23)$ & $150(150)$ \\
\hline$B$ résultats & $-1(85)$ & $+1(91)$ & $+4(79)$ & $-2(65)$ & $320(320)$ \\
\hline$A B$ résultats & $-9(25)$ & $-6(29)$ & $+10(41)$ & $+7(35)$ & $130(130)$ \\
\hline résultats moyens & $+0(7)$ & $+0(7)$ & $+1(8)$ & $+0(6)$ & $28(28)$ \\
\hline résultats insuffisants & $-5(6)$ & $-7(5)$ & $+2(13)$ & $+11(21)$ & 45 ( 45) \\
\hline \begin{tabular}{|l} 
TOTAL \\
\end{tabular} & $204(204)$ & $211(211)$ & $177(177)$ & $160(160)$ & $752(752)$ \\
\hline
\end{tabular}

Lecture : les chiffres entre parenthèses sont les effectifs observés, les chiffres hors parenthèses précédés d'un signe +/- sont les écarts aux effectifs théoriques (calculés par des tables de contingence). Les encadrements sont les plus fortes contributions au chi2 (test statistique utilisé), c'est-à-dire les plus forts écarts entre effectifs réels et théoriques.

Les ND sont significativement sur-représentés parmi les lycéens dont les résultats sont jugés « insuffisants ». Au contraire, les TM et AND sont sur-représentés parmi les élèves reconnus pour leur excellence, les TF parmi ceux qui obtiennent $d^{\prime}$ '« assez bons résultats ». Cette étude catégorie par catégorie indiquerait les modèles du bon, de l'excellent et du mauvais élèves. Quel bilan établir de ces interprétations ?

Les élèves endossant les traits masculins représenteraient, dans les croyances des enseignants d'EPS, l'excellence dans leur discipline. Cette partie du corps professoral (en particulier l'enseignante de notre échantillon, avec qui TF et ND ont les moins bonnes notes) reconnaîtrait moins aisément l'excellence des élèves qui rejettent les traits masculins, tout particulièrement les TF, car cette situation n'est pas conforme à leurs représentations. II s'agirait de phénomènes de dissonance cognitive (Festinger, 1957, cité par Bienaimé-Patinet, 2009) ; les individus ont tendance à rejeter, par minimisation ou déni, les évènements entrant en contradiction avec leurs croyances et modes de pensée. L'élève ND représenterait le contre-modèle en EPS. Tout d'abord, il est celui dont la probabilité est la plus forte d'être caractérisé par des résultats insuffisants, même lorsqu'il 


\section{eJRIEPS 22 janvier 2011}

fournit des efforts, dont on souligne les progrès, façon courtoise d'adoucir ses difficultés. Sa réussite semble également plus facilement nuancée, ce qui est une façon pour l'enseignant de marquer les limites de sa satisfaction (Felouzis, 1997). Ensuite, les descriptions de sa personne - ses qualités - apparaissant sur les bulletins sont plus négatives que celles de ses camarades, tout particulièrement les TM. Enfin, ils sont ceux à qui l'on demande plus de «persévérer », tout en étant ceux qui déçoivent le plus les enseignants. Cette demande de persévérance sous-tendrait de moindres qualités dont disposeraient les élèves ND pour réussir en EPS. Par conséquent, ils devraient fournir des efforts supplémentaires pour obtenir les mêmes résultats que les exemplaires TM ou AND. Si les derniers cités sont, semble-t-il, les lycéens avec qui les enseignants d'EPS entretiennent les relations les plus agréables, ce sont les TM qui représenteraient le mieux les élèves possédant les qualités psychologiques, les «brillants». En outre, les enseignants d'EPS attendraient plus des élèves, garçons ou filles, qui endossent les traits masculins.

Certaines croyances sexistes, car elles sous-tendent une supériorité des garçons, persisteraient dans le corps professoral. Les garçons semblent apparaître comme plus « doués », ayant de plus grandes facilités pour réussir en EPS et y atteindre l'excellence. Par conséquent, les filles auraient à fournir plus d'efforts pour rejoindre les garçons sur le chemin de la réussite et doivent les dépasser pour être reconnues comme excellentes ou pour leurs qualités motrices, leurs « dons».

Les garçons apparaissent comme le modèle à suivre, l'élève qui réussit sans effort car «talentueux ». Ils semblent très à l'aise dans leur métier d'élève en EPS, discipline dans laquelle les enseignants seraient, en général, plus exigeants envers eux. Une étude de Cogérino et Lachelard (2003, citées par Bienaimé-Patinet, 2009) montre que les enseignants d'EPS perçoivent les élèves au travers de critères sexués. Cette double lecture n'est pas limitée à l'EPS.

En effet, le conseil de classe attendrait plus des garçons vis-à-vis des résultats, mais serait plus exigeant envers les filles au niveau du comportement et du travail à fournir. L'idée d'une certaine supériorité des garçons subsisterait: meilleurs résultats, moins d'efforts pour réussir, plus fort potentiel et explication des mauvais résultats par un manque de travail. Face à ces sous-réalisateurs, les filles devraient fournir plus d'efforts, persévérer pour réussir. Leur échec ne s'expliquerait pas par le manque de travail. Les efforts fournis et leur bonne volonté sont souvent soulignés. Ainsi, les appréciations « travaille mais ne réussit pas », qui semblent assez positives sont riches d'un double 


\section{eJRIEPS 22 janvier 2011}

sens. Si la réussite n'est pas là malgré les efforts, c'est qu'il manquerait quelque chose. Bien que filles et garçons de notre échantillon ont une moyenne générale équivalente, les professeurs auraient des attentes sexuées. L'effet du genre est encore plus fort. Les ND seraient le contre-modèle, ils ont de plus fortes probabilités d'obtenir des résultats jugés insuffisants, d'être éloignés de la morale scolaire, de moins recevoir de distinctions que leurs camarades. Au contraire, les TM tiendraient le rôle de l'élève efficace dont les résultats sont salués, sans pour autant qu'il soit un élève docile. Cet élève efficace reçoit plus facilement les plus hautes distinctions du conseil de classe : les félicitations et la reconnaissance de son excellence.

La féminité des élèves les stabiliserait dans la norme. L'orientation de genre des AND ou des TF n'a pas d'effet sur les appréciations générales. Ils se répartissent comme l'ensemble des lycéens. L'institution scolaire considérerait ces élèves comme les lycéens normaux. Si elle attend plus de leur part que de celle des ND, c'est envers les TM qu'elle est la plus exigeante. Ce degré d'exigence pourrait expliquer, par un effet Pygmalion, les meilleurs résultats des TM.

\section{Conclusion}

L'idée que l'institution scolaire serait favorable au genre féminin est souvent avancée pour expliquer les différences de réussite scolaire entre filles et garçons; notre Ecole serait plus proche des valeurs « féminines ». Nos résultats semblent montrer que ce n'est pas le cas, au moins dans l'établissement étudié. Malgré la plus forte proportion des filles (environ trois pour deux garçons), ce sont les TM qui ont une plus forte probabilité empirique d'obtenir une meilleure moyenne générale que leurs camarades, mais aussi des résultats supérieurs en mathématiques et histoire-géographie. Nous avons observé les notes dans ces deux disciplines, ainsi qu'en français et en philosophie (Moniotte, 2010). Dans celles-ci, ce sont les filles TM qui ont des moyennes significativement élevées. Les TM sont également plus facilement reconnus comme des lycéens excellents et concentreraient de plus fortes attentes professorales.

L'EPS, discipline dans laquelle les garçons réussissent mieux que les filles, serait un révélateur de la domination du genre masculin - les traits et rôles masculins seraient scolairement plus rentables - dans l'institution étudiée. Il existerait un effet loupe de l'EPS. Elle est une discipline d'enseignement à part entière, porteuse d'un curriculum caché masculiniste, déjà dénoncé par Mosconi (1994) au sein de l'institution scolaire. L'EPS se pose certaines questions qui méritent d'être posées au système scolaire dans sa globalité. 


\section{eJRIEPS 22 janvier 2011}

Quelle place les pratiques extra-scolaires prennent sur la scolarité des élèves? L'importance en EPS des pratiques physiques fédérales est souvent soulevée. D'ailleurs, une des difficultés des enseignants est d'évaluer ce qui a été enseigné pendant les leçons d'EPS et non pas au sein des clubs.

Nous pouvons également nous interroger sur la légitimité de la domination de la culture sportive en EPS. II existe, en effet, bien d'autres formes de cultures corporelles transmissibles à travers les nombreuses APSA disponibles. Cependant, en s'éloignant de la culture sportive, l'EPS pourrait également s'éloigner de celle de l'Ecole. En effet, malgré les discours prônant la réussite de tous, l'institution scolaire produit des classements interélèves, des hiérarchies inter-filières et organise une compétition tout au long du cursus scolaire. Les diplômes qu'elle délivre servent de titres pour se positionner dans la hiérarchie sociale. « La note transmet essentiellement une information sur le classement relatif des élèves ; elle désigne les «bons » et les «mauvais ». Elle s'inscrit donc dans un contexte de compétition, non seulement sportive mais vitale, puisque la sélection à l'entrée des études longues décide de façon quasi définitive de la carrière de l'enfant. Son appartenance aux classes dirigeantes ou dirigées en découle, avec tous les privilèges ou toutes les frustrations qui les accompagnent »(Cardinet, 1986, p. 15). Si l'appartenance à une classe sociale découle de la carrière scolaire, ce qui est discutable, le problème principal provient plutôt du poids de l'origine sociale sur la scolarité des élèves. Bourdieu et Passeron (1970) ont dénoncé ce processus de reproduction. II s'explique en partie par la connivence culturelle entre l'Ecole et les classes aisées. Nos résultats montreraient aussi une connivence avec le genre masculin - puisque les typés masculins ont objectivement plus de chances d'être félicités et reconnus comme excellents par le conseil de classe. II semble que dans l'établissement étudié, les traits masculins ne soient pas seulement plus rentables en EPS.

\section{Bibliographie}

Baillette, F., \& Liotard, P. (1999). Sport et virilisme. Montpellier : Quasimodo \& fils.

Bem, S. (1978). Au-delà de l'androgynie. Quelques préceptes osés pour une identité de sexe libérée in M.-C. Hurtig \& M.-F. Pichevin (Eds. and Trans.) (1986), La différence des sexes. Questions de psychologie (251-270). Paris : Tierce. 


\section{eJRIEPS 22 janvier 2011}

Bienaimé-Patinet, C. (2009). Équité sexuée et centrations attentionnelles des enseignants d'éducation physique et sportive en contexte mixte. Thèse de doctorat non publiée, Université de Picardie, Amiens.

Bourdieu, P., \& Passeron, J.-C. (1970). La reproduction. Paris : Minuit.

Caillat, M. (2002). Le sport. Paris : Le Cavalier bleu.

Cardinet, J. (1986). Evaluation scolaire et pratique. Bruxelles : De Boeck.

Chalabaev, A. (2006). L'influence des stéréotypes sexués sur la performance et la motivation en sport et en éducation physique et sportive. Thèse de doctorat non publiée, Université Fourier, Grenoble.

Combaz, G. (1991). EPS : la mixité : opinions et souhaits des élèves, EPS, 231, 62-65.

Constantinople, A. (1973). Masculinité/féminité. Exception à un célèbre adage, in M.-C. Hurtig \& M.-F. Pichevin (Eds. and Trans.)(1986), La différence des sexes. Questions de psychologie (225-250). Paris : Tierce.

David, B. (2000). Education physique et sportive. La certification au baccalauréat. France : INRP.

Davisse, A., \& Louveau, C., (2003). Sports, école, société : la différence des sexes. Féminin, masculin et activités sportives. Paris : l'Harmattan.

Detrez, C. (2004). Une représentation différentielle du corps de l'homme et de la femme, Problèmes politiques et sociaux, 907, 34-35.

Duru-Bellat, M. (1994). Filles et garçons à l'école, approches psychologiques et psychosociales. Note de synthèse, Revue française de pédagogie, 110, 111-141.

Duru-Bellat, M. (1995a). Filles et garçons à l'école, approches psychologiques et psychosociales. Note de synthèse, Revue française de pédagogie, 111, 75-109.

Duru-Bellat, M. (1995b). Une facette implicite du métier d'élève, in M. De Manassein (Ed.), De l'égalité des sexes (183-202). Paris : CNDP.

Duru-Bellat, M. (2004a). La (re)production des rapports sociaux de sexe : quelle place pour l'institution scolaire?, Travail! genre et sociétés, 19, 131-149.

Duru-Bellat, M. (2004b). L'école des filles. Quelle formation pour quels rôles sociaux? Paris : l'Harmattan.

Felouzis, G. (1993). Interactions en classe et réussite scolaire. Une analyse des différences filles-garçons. Revue française de sociologie, 34(2), 199-222.

Felouzis, G. (1994). Le collège au quotidien. Paris : PUF.

Felouzis, G. (1997). L'efficacité des enseignants. Paris : PUF. 


\section{eJRIEPS 22 janvier 2011}

Ferez, S. (2004). De l'expression corporelle aux Activités Physiques Artistiques (A.P.A) : Subversion sexuée et différenciation sexuée en Education Physique et Sportive (E.P.S), STAPS, 66, 113-128.

Fontayne, P., Sarrazin, P., \& Famose, J.-P. (2000). The Bem Sex-Role Inventory: Validation of a short-version for French teenagers, European Review of Applied Psychology, 50(4), 405-417.

Fontayne, P., Sarrazin, P., \& Famose, J.-P. (2001). Les pratiques sportives des adolescents : une différenciation selon le genre. STAPS, 55, 23-37.

Forquin, J.-C. (1989). Ecole et culture. Le point de vue des sociologies britanniques. Bruxelles : De Boeck.

Goffman, E. (2002). L'arrangement des sexes. Paris : La Dispute.

Hurtig, M.-C., Kail, M. \& Rouch, H. (Eds.). (2002). Sexe et genre. Paris : CNRS.

Laugier, S. (2004). Performativité, normativité et droit, Archives de philosophie, 67, 607627.

Lefevre, B. (2005). Pratiques sportives et mises en scène du genre : entre conservation, lissage et reformulation. L'exemple de la jupe chez les joueuses de tennis, in T. Terret (Ed.), Sport et genre. Volume 1. La conquête d'une citadelle masculine (369-379). Paris : L'Harmattan.

Marro, C., \& Vouillot, F. (2004). Quelques concepts clés pour penser et former à la mixité, Carrefours de l'éducation, 17, 3-21.

Mathieu, N.-C. (2002). Les transgressions de sexe et de genre à la lumière de donnés ethnographiques in M.-C. Hurtig, M. Kail \& H. Rouch (Eds.), Sexe et genre (6980). Paris : CNRS.

MEN( 2007). Image du sport scolaire et pratiques d'enseignement au collège et au lycée 2005-2006. Paris : CNDP.

Merle, P. (1996). L'évaluation des élèves. Enquête sur le jugement professoral. Paris: PUF.

Moniotte, J. (2010). La domination masculine en EPS. L'analyse des mécanismes du jugement professoral en éducation physique et sportive. Thèse de doctorat non publiée, Université de Franche-Comté, Besançon.

Mosconi, N. (1994). Femmes et savoir; la société, l'école et la division sexuelle des savoirs. Paris : L'harmattan.

Mosconi, N. (1995). La mixité scolaire: une institution masculiniste, Les Cahiers du MAGE, 1, 17-24. 
Mosconi, N. (2004). Effets et limites de la mixité scolaire, Travail, genre et société, 11, 165-174.

Nazé, Y. (2006). L'astronomie au féminin. Paris : Vuilbert.

Perrenoud, P. (1998). L'évaluation des élèves. De la fabrication de l'excellence à la régulation des apprentissages. Bruxelles: De Boeck.

Poggi, M.-P. (2000). Quelles pratiques corporelles au collège? L'EPS sous l'angle de la sociologie des parcours scolaires. Thèse de doctorat non publiée, université René Descartes, Paris 5.

Sarrazy, B. (2000). Les bulletins scolaires ne servent-ils qu'à évaluer les compétences des élèves? Contribution à l'analyse des fonctions didactique et pédagogique des appréciations, Les sciences de l'éducation, 33, 51-77.

Terret, T., Cogérino, G., \& Rogowski, I. (2006). Pratiques et représentations de la mixité en EPS, Paris, Revue EPS.

Vidal, J.-P. (2008). De la déconstruction de la différence des sexes à la «neutralisation des sexes », pour une société « postsexuelle »!, Connexions, 90, 123-138.

Vigarello, G. (1978). Le corps redressé. Paris : Armand Colin.

Vigneron, C. (2004). La construction des inégalités de réussite en EP au baccalauréat entre filles et garçons. Thèse de doctorat non publiée, Université de Bourgogne, Dijon.

Vinet, N. (2008). Émergence, perspective et mise à l'épreuve contemporaine du constructivisme sexué, Connexions, 90, 57-75.

Williamson, K.M. (1996). Gender issues. In S.J Silverman \& C.D. Ennis (Eds.), Student Learning in Physical Education, (pp. 80-100). USA : Human kinetics. 